\title{
The clinical profiles and outcomes of HIV- negative cryptococcal meningitis patients in type II diabetes mellitus
}

Hang $\mathrm{Li}^{1,2+}$, Xiaojing $\mathrm{Li}^{3 \dagger}$, Lei Zhang ${ }^{1,2 \dagger}$, Wenjie Fang ${ }^{1,2}$, Keming Zhang ${ }^{1,2}$, Amir Arastehfar ${ }^{4}$, Macit Ilkit ${ }^{5}$,

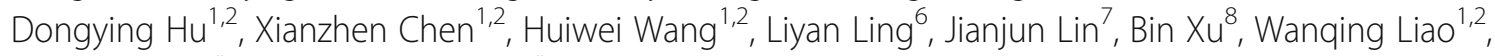
Weihua Pan ${ }^{1,2^{*}}$ and Qilong Zhang ${ }^{8,9^{*}}$

\begin{abstract}
Background: The clinical profiles and outcomes of cryptococcal meningitis have been shown to vary depending on the underlying condition. The aim of this study was to investigate clinical characteristics and outcomes in patients with and without type II diabetes mellitus.

Methods: A retrospective study was performed. Clinical data of HIV-negative cryptococcal meningitis patients with type II diabetes mellitus $(n=26)$ and without type II diabetes mellitus $(n=52)$ referring to the Jiangxi Chest Hospital between January 2012 to December 2018 were analyzed. The data were analyzed using chi square, noneparametric tests, and logistic regression. P-values $<0.05$ were considered significant.

Results: In this study, cryptococcal meningitis patients suffering from type II diabetes mellitus had a higher mortality ( $23.08 \%$ vs. $7.69 \% ; P=0.055)$, and required longer hospitalization (59.58 vs. 42.88 days; $P=0.132$ ). Moreover, cerebrospinal fluid examinations revealed that cryptococcal meningitis patients with type II diabetes mellitus had higher opening pressure (271.54 vs. $\left.234.23 \mathrm{mmH}_{2} \mathrm{O} ; P=0.125\right)$. The results of multivariate regression analysis revealed that cryptococcal meningitis patients with type II diabetes were more often presented with visual disorders (28.54\% vs. 11.54\%; [95\% Cl 0.056-0.705]; $p=0.012$ ), and had higher cerebrospinal fluid protein levels (1027.62 \pm 594.16 vs. $705.72 \pm 373.88 \mathrm{mg} / \mathrm{l} ;$ [95\% Cl 1.000-1.002]; $p=0.016$ ). Among patients with type II diabetes mellitus, nausea and vomiting was more frequent at the initial visit in those died (100\% vs. $50 \% ; p=0.027$ ), and $66 \%$ of died type II diabetes mellitus patients were poorly controlled blood glucose level, compared with $30 \%$ in survival type II diabetes mellitus patients.

Conclusion: This study suggests that cryptococcal meningitis patients with type II diabetes mellitus differ significantly from cryptococcal meningitis patients without type II diabetes mellitus with respect to clinical symptoms such as visual disorders and cerebrospinal fluid examination. The presence of nausea and vomiting among type II diabetes mellitus patients could have implication in mortality.
\end{abstract}

Keywords: Cryptococcal meningitis, Type II diabetes mellitus, Clinical profile, Prognosis

\footnotetext{
*Correspondence: panweihua9@sina.com; qilong681015@126.com

${ }^{\dagger}$ Hang Li, Xiaojing Li and Lei Zhang contributed equally to this work.

'Shanghai Key Laboratory of Molecular Medical Mycology, Shanghai

Changzheng Hospital, Second Military Medical University, Shanghai 200003,

P. R. China

${ }^{8}$ Department of Neurology, Jiangxi Chest Hospital, Jiangxi 330000, P. R. China

Full list of author information is available at the end of the article
}

(c) The Author(s). 2021 Open Access This article is licensed under a Creative Commons Attribution 4.0 International License, which permits use, sharing, adaptation, distribution and reproduction in any medium or format, as long as you give appropriate credit to the original author(s) and the source, provide a link to the Creative Commons licence, and indicate if changes were made. The images or other third party material in this article are included in the article's Creative Commons licence, unless indicated otherwise in a credit line to the material. If material is not included in the article's Creative Commons licence and your intended use is not permitted by statutory regulation or exceeds the permitted use, you will need to obtain permission directly from the copyright holder. To view a copy of this licence, visit http://creativecommons.org/licenses/by/4.0/ The Creative Commons Public Domain Dedication waiver (http://creativecommons.org/publicdomain/zero/1.0/) applies to the data made available in this article, unless otherwise stated in a credit line to the data. 


\section{Background}

Cryptococcal meningitis (CM) is a serious central nervous system infection caused by Cryptococcus neoformans and Cryptococcus gattii complexes [1], and cryptococcal meningitis is a leading cause of death among HIV-infected individuals, especially in SubSaharan Africa [2, 3]. Risk factors for cryptococcal infection include: HIV infection, transplant recipients, malignancy, and corticosteroid use. Due to the introduction of effective antifungal drugs and improved management of HIV, the incidence and mortality of cryptococcal meningitis in HIV-infected patients has decreased in recent years. However, the increasing population of HIVnegative patients with immunocompromised conditions has received increasing attention [4-7]. Importantly, approximately $80 \%$ of cryptococcal meningitis cases in China and the US were reported in HIV-negative populations [8, 9]. Many of these patients have underlying diseases, such as cancer, systemic lupus erythematosus, type II diabetes mellitus (DM II), nephrotic syndrome and tuberculosis [4-7]. Of note, the impact of various clinical features on survival of cryptococcal meningitis in HIV-infected individuals have been adequately described in previous studies [10, 11]. Moreover, some studies have investigated the clinical presentation and therapeutic outcomes in HIV-uninfected populations [6, 12], but comparative assessments of cryptococcal meningitis patients with and without underlying diseases are limited.

Type II diabetes mellitus is a group of metabolic disorders characterized by a long-term elevation of blood glucose levels. China has the world's largest type II diabetes mellitus population; according to the latest national survey, 11.6 and $50.1 \%$ of Chinese adults have diabetes and prediabetes disorders, respectively, accounting for nearly $25 \%$ of the global diabetes population [13, 14]. Inadequate glucose management in diabetic patients increases their risk of infections and usually requires more hospitalization compared with that of the general population [14-16]. The prevalence of diabetes mellitus in cryptococcosis patients varied from 2 to $25 \%$, based on previous studies, and the diagnosis of diabetes mellitus was also associated with 1-year and overall mortality in cryptococcosis populations [1719]; therefore, understanding the differences of clinical manifestations between cryptococcal meningitis patients with and without type II diabetes mellitus may have clinical implications and contribute in ameliorating survival rate. Consequently, we conducted a retrospective study and evaluated the clinical profiles of HIV-negative cryptococcal meningitis patients with and without type II diabetes mellitus to identify factors holding prognostic values and implicated in mortality.

\section{Methods}

This study protocol was approved by the Institutional Review Board of the Jiangxi Chest Hospital (no. 20190304). Informed consent was waived by the Institutional Review Board due to the retrospective nature of the study design.

\section{Study setting \& participants}

We retrospectively reviewed the medical records of patients with cryptococcal meningitis admitted to the Department of Internal Neurology, Jiangxi Chest Hospital. Between January 2012 and December 2018, HIVnegative cryptococcal meningitis patients with type II diabetes mellitus ( $n=26$, DM II group) and without type II diabetes mellitus ( $n=52$, non-DM II group) were enrolled. The inclusion criteria are including: confirmed cryptococcal meningitis patient with type II diabetes mellitus and without type II diabetes mellitus; Follow-up data were available. Patients receiving antifungal treatments prior to admission, those presenting recurrent cryptococcal meningitis, and aged $<18$ years old were excluded from our study. The diagnosis of cryptococcal meningitis was based on positive results of cerebrospinal fluid culture, cerebrospinal fluid cryptococcal antigen test (IMMY, OK, USA), or positive cerebrospinal fluid India ink staining. Type II diabetes mellitus was defined according to the Chinese guidelines for diabetes mellitus: (1) random blood sugar test: a blood sugar level is 200 mg per deciliter $(\mathrm{mg} / \mathrm{dL})$ - 11.1 millimoles per liter (mmol/L)- or higher; (2) fasting blood sugar: a fasting blood sugar is $7 \mathrm{mmol} / \mathrm{L}$ or higher on two separate tests; (3) oral glucose tolerance test: a blood sugar level is

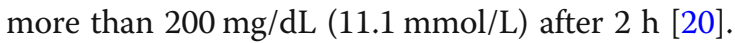

\section{Data collection}

For each patient, the following data were obtained: demographic information, sex, location, time of cryptococcal meningitis symptom emergence, time of cryptococcal meningitis confirmation, underlying diseases except type II diabetes mellitus, duration of type II diabetes mellitus course, antifungal regimen, clinical manifestations, laboratory findings, brain image, length of hospital stay (days) and outcome. Temporary drains, lumbar drains, or external ventricular drains for the control of increased intracranial pressure were also recorded. Brain image abnormalities were defined according to head computed tomography or magnetic resonance imaging scans as any parenchymal abnormality (including meningeal enhancement, bleeding, and strokes), and discharge data were used to determine the outcome. Two authors collected the clinical data, and these data were checked by the third author. Disagreements were resolved by discussion between two authors supervised by an expert. 


\section{Statistical analysis}

IBM SPSS (version 20, International Business Machines Corporation, New York, NY, USA) was used for statistical analysis, and the categorical data were reported as the means \pm standard deviations or percentages. Comparisons between groups were performed by the chisquare test, Mann-Whitney $U$ test and logistic regression. $P$-values less than 0.05 were considered statistically significant.

\section{Results}

\section{Initial characteristics and laboratory findings}

The demographic details, initial clinical symptoms and laboratory data of patients with and without type II diabetes mellitus are shown in Table 1 . Hypertension was the most common in the type II diabetes mellitus group, while in the non-type II diabetes mellitus group, respiratory disease was most common. There was no significant difference in age and underlying diseases between the two groups. We also found no significant difference in the initial clinical symptoms between the type II diabetes mellitus and the non- type II diabetes mellitus group, except for visual disorders, which were more frequent in the latter group (28.54\% vs. $11.54 \%, p=0.038)$. As for the type of visual disorders, the most common between the two groups was blurred vision, accounting for $62.5 \%(n=5)$ and $80 \%(n=$ 4) in the type II diabetes mellitus group and the non- type II diabetes mellitus group, respectively. Other visual impairment in type II diabetes group, including blindness $(n=1)$, the abduction of eye limited $(n=1)$ and diplo$\operatorname{pia}(n=1)$. Diplopia occurred in one patients in non-type II diabetes groups. The most common symptoms in the two groups were headache (96.15\%), followed by fever (76.92\%), nausea/vomiting (52.56\%), conscious disturbance (21.79\%), and visual disorders (15.38\%).

With regard to the cerebrospinal fluid examinations, the type II diabetes mellitus group had a higher cerebrospinal fluid protein level $(1027.62 \pm 594.16 \mathrm{mg} / \mathrm{l})$ than the non-type II diabetes group $(705.72 \pm 373.88 \mathrm{mg} / \mathrm{l}$; [95\% CI 1.000,1.002]; $p=0.016$ ). There was no significant difference in opening pressure between the two groups (mean 271.54 vs. $234.23 \mathrm{mmH}_{2} \mathrm{O} ; p=0.125$ ). There was no significant difference in brain images between the two groups. Meningeal enhancement was the most common manifestation between two groups, which responsible for $38.46 \%$ in type II diabetes mellitus group and $42.30 \%$ in non-type II diabetes group, respectively. Among 78 patients, 49 patients were diagnosed based on culture, 39 patients were diagnosed based on culture cryptococcal antigen and positive India ink stain.

\section{Treatment and outcomes}

All the patients were treated with systemic antifungal drugs, as shown in Table 2. The induction treatment included amphotericin B with flucytosine, lipid amphotericin B infusion with flucytosine, amphotericin B + fluconazole, and voriconazole \pm flucytosine, there were no significant differences between the patients in the two groups who received each regimen. For the management of increased intracranial pressure, most patients received repeated lumbar puncture, and a temporary drain was administered in one type II diabetes mellitus patient and two without type II diabetes patients. For long-term cerebrospinal fluid drainage, permanent shunt devices were inserted in one type II diabetes mellitus patient and three non-type II diabetes group patients. The time of hospitalization in the type II diabetes mellitus group was longer than that in the non- type II diabetes mellitus group (59.58 vs. 42.88 days, $p=0.132$, respectively). The death rate was higher in the type II diabetes mellitus group $(6 / 26 ; 23.08 \%)$ than in the non-type II diabetes mellitus group $(4 / 52 ; 7.69 \%, p=0.055)$.

We also analyzed possible factors associated with mortality between the surviving and dead cryptococcal meningitis patients with type II diabetes mellitus (Table 3). We found that at the initial presentation, nausea and vomiting was associated with mortality among type II diabetes mellitus patients $(p=0.027)$. Although poor type II diabetes mellitus management was not associated with mortality, $66 \%$ of patients who died did not manage diabetes well, compared with $30 \%$ in surviving patients.

\section{Discussion}

Cryptococcal meningitis is a common opportunistic central nervous system infection that can occur in both immunocompromised and immunocompetent patients [21]. Many underlying diseases and conditions, such as HIV infection, organ transplantation, autoimmune disease, chronic kidney disease, and long-term steroid use, are reported as risk factors for Cryptococcus infection [4, $5,11,22]$. Thus, understanding the clinical characteristics of cryptococcal meningitis patients suffering from secondary complications is important for clinical management and reducing mortality.

In this study, we found that cryptococcal meningitis patients with type II diabetes mellitus were older and more often presented with visual disorders when compared to cryptococcal meningitis patients without type II diabetes mellitus. In cerebrospinal fluid examinations, more patients had higher opening pressure and cerebrospinal fluid protein levels. In addition, the time of hospitalization was longer and mortality was higher in the type II diabetes mellitus group than in the non-type II diabetes mellitus group.

In both groups, the most common symptoms of the 78 patients were fever, headache, nausea, vomiting and consciousness disturbance, which were partially consistent with data reported in previous studies [3]. There 
Table 1 Univariate statistics and multivariate logistic regression analysis of patients' characteristics and laboratory findings on admission

\begin{tabular}{|c|c|c|c|c|c|}
\hline \multirow[t]{2}{*}{ Characteristics } & \multicolumn{3}{|l|}{ Univariate analysis } & \multicolumn{2}{|c|}{ Multivariate analysis } \\
\hline & DM II group $(n=26)$ & Non-DM II group $(n=52)$ & $P$ value & $(95 \% \mathrm{Cl})$ & $P$ value \\
\hline Age (years) & $57.31 \pm 14.27$ & $52.20 \pm 15.84$ & 0.173 & & \\
\hline Sex: Male (n \%) & $12(46.15 \%)$ & $29(55.77 \%)$ & 0.423 & & \\
\hline Rural area (n \%) & $21(80.77 \%)$ & $44(84.61 \%)$ & 0.667 & & \\
\hline \multicolumn{6}{|l|}{ Underlying conditions } \\
\hline Respiratory diseases (n \%) & $3(11.53 \%)$ & $7(13.46 \%)$ & 0.811 & & \\
\hline Chronic kidney diseases (n \%) & $2(7.69 \%)$ & $4(7.69 \%)$ & 1.000 & & \\
\hline Hepatitis B (n \%) & $3(11.53 \%)$ & $3(5.77 \%)$ & 0.367 & & \\
\hline Hypertension (n \%) & $4(15.38 \%)$ & $2(3.85 \%)$ & 0.071 & $(0.026,1.109)$ & 0.064 \\
\hline Autoimmune disease (n \%) & $3(11.53 \%)$ & $4(7.69 \%)$ & 0.575 & & \\
\hline Hematology and oncology (n \%) & 0 & $1(1.92 \%)$ & 0.477 & & \\
\hline The duration before confirmed diagnosis (days) & $27.00 \pm 23.13$ & $26.30 \pm 18.93$ & 0.501 & & \\
\hline Duration of DM II course (years) & $4.20 \pm 3.34$ & - & - & & \\
\hline \multicolumn{6}{|l|}{ Clinical parameters } \\
\hline Fever (n \%) & $21(80.77 \%)$ & $39(75.00 \%)$ & 0.569 & & \\
\hline Headache (n \%) & 25(96.15\%) & $50(96.15 \%)$ & 1.000 & & \\
\hline Nausea/Nomiting (n \%) & $16(61.54 \%)$ & $25(48.07 \%)$ & 0.262 & & \\
\hline Vision disorders (n \%) & $8(28.54 \%)$ & $6(11.54 \%)$ & $0.038^{*}$ & $(0.056,0.705)$ & $0.012^{*}$ \\
\hline Auditory symptoms (n \%) & $2(7.69 \%)$ & $4(7.69 \%)$ & 1.000 & & \\
\hline Seizure (n \%) & $3(11.54 \%)$ & $3(5.77 \%)$ & 0.367 & & \\
\hline Conscious disturbance (n \%) & $8(30.77 \%)$ & $9(17.31 \%)$ & 0.175 & & \\
\hline \multicolumn{6}{|l|}{ CSF examinations } \\
\hline Opening pressure $\left(\mathrm{mmH}_{2} \mathrm{O}\right)$ & $271.54 \pm 100.78$ & $234.23 \pm 105.98$ & 0.125 & & \\
\hline CSF protein (mg/l) & $1027.62 \pm 594.16$ & $705.72 \pm 373.88$ & $0.031^{*}$ & $(1.000,1.002)$ & $0.016^{*}$ \\
\hline CSF glucose (mmol/l) & $2.08 \pm 1.01$ & $2.30 \pm 1.41$ & 0.250 & & \\
\hline Positive India ink stain (n \%) & 19(73.08\%) & $40(76.92 \%)$ & 0.709 & & \\
\hline Positive cryptococcal antigen (n \%) & 25(96.15\%) & $50(96.13 \%)$ & 1.000 & & \\
\hline Positive culture result (n \%) & $17(65.38 \%)$ & $32(61.54 \%)$ & 0.740 & & \\
\hline \multicolumn{6}{|l|}{ Brain images (CT or MRI) } \\
\hline Meningeal enhancement (n \%) & $10(38.46 \%)$ & $22(42.30 \%)$ & 0.745 & & \\
\hline Hydrocephalus (n \%) & $2(7.69 \%)$ & $4(7.69 \%)$ & 1.000 & & \\
\hline Cerebral infarction (n \%) & $4(15.38 \%)$ & $6(11.54 \%)$ & 0.632 & & \\
\hline Abscess (n \%) & $2(7.69 \%)$ & 2(3.84\%) & 0.468 & & \\
\hline Normale $^{a}$ (n \%) & $8(30.77 \%)$ & $18(34.62 \%)$ & 0.734 & & \\
\hline
\end{tabular}

DM II diabetes mellitus II, CSF cerebrospinal fluid, CT computed tomography, MRI magnetic resonance imaging, Cl confidence interval ${ }^{a}$ Normal brain images on CT or MRI were defined as no obvious intracranial lesions demonstrated; ${ }^{*} p<0.05$

were no significant differences in the above mentioned symptoms between both of the groups, with the exception of vision disorders. The reasons for vision disorders include: diabetes itself can cause visual impairment, some patients have visual impairment in the diabetic stage but worsen after cryptococcal meningitis, and the other is simply caused by cryptococcal meningitis. Considering that diabetes itself can cause visual disturbance, perhaps this is the main reason for the difference between two groups [23]. In addition, we found that type II diabetes mellitus patients presented with higher intracranial pressure. Although higher intracranial pressure has been associated with mortality among cryptococcal meningitis patients [24, 25], we did not observe a direct relationship between intracranial pressure and mortality in the present study, probably because a ventriculoperitoneal shunt or 
Table 2 Treatment and clinical outcomes of the two groups

\begin{tabular}{llll}
\hline Variables & DM II group $(\boldsymbol{N}=\mathbf{2 6})$ & Non-DM II group $(\boldsymbol{N}=\mathbf{5 2})$ & $\boldsymbol{P}$ value \\
\hline Treatment & & & \\
$\mathrm{AMB}+5 \mathrm{FC}(\mathrm{n} \%)$ & $18(69.23 \%)$ & $41(78.85 \%)$ & 0.351 \\
$\mathrm{Lip} \mathrm{AMB}+5 \mathrm{FC}(\mathrm{n} \%)$ & $5(19.23 \%)$ & $6(11.54 \%)$ & 0.284 \\
$\mathrm{AMB}+\mathrm{FCZ}(\mathrm{n} \%)$ & 0 & $3(5.77 \%)$ & $2(3.84 \%)$ \\
VCZ $\pm 5 F C(\mathrm{n} \%)$ & $3(11.54 \%)$ & $47(90.38 \%)$ & 0.212 \\
Treatment for IICP $(\mathrm{n} \%)$ & $24(92.30 \%)$ & $39(75.00 \%)$ & 0.191 \\
Repeated LP $(\mathrm{n} \%)$ & $20(76.92 \%)$ & $5(9.62 \%)$ & 0.779 \\
Temporary drain (n \%) & $2(7.69 \%)$ & $2(3.85 \%)$ & 0.852 \\
Permanent shunt device (n \%) & $1(3.85 \%)$ & $42.88 \pm 1791$ & 0.779 \\
Length of hospitalization (days) & $59.58 \pm 29.64$ & $4(7.69 \%)$ & 0.132 \\
In-hospital mortality & $6(23.08 \%)$ & 0.055 \\
\hline
\end{tabular}

Abbreviations: $D M$ // type II diabetes mellitus, $A M B$ amphotericin $B, 5 F C$ flucytosine, Lip $A M B$ lipid amphotericin $B, F C Z$ fluconazole, VCZ voriconazole, IICP increased intracranial pressure, $L P$ lumbar puncture

temporary drain was used in patients with intracranial pressure or because mannitol is used as medication to reduce intracranial pressure.

Among the included 78 cryptococcal meningitis cases, $75 \%$ patients $(n=59)$ received standard antifungal therapy. The antifungal regimen in the two groups was not significantly different. Five patients in the two groups were treated with voriconazole combined with flucytosine or voriconazole alone and achieved good results. Voriconazole has been found to be an effective drug for cryptococcal meningitis in some studies [26, 27]. Although requires larger patient population, our study showed that voriconazole in combination with flucytosine might be a useful alternative therapy for cryptococcal meningitis patients.

Table 3 Possible risk factors associated with mortality among CM patients with DM II

\begin{tabular}{|c|c|c|c|}
\hline Variables & Surviving patients $(N=20)$ & Dead patients $(N=6)$ & $P$ value \\
\hline Age (years) & $57.95 \pm 15.38$ & $55.75 \pm 10.61$ & 0.533 \\
\hline Sex: Male (n \%) & $9(45.00 \%)$ & $3(50.00 \%)$ & 0.829 \\
\hline Duration before confirmed diagnosis (days) & $29.85 \pm 22.80$ & $17.83 \pm 11.05$ & 0.355 \\
\hline Duration of DM II course (years) & $4.27 \pm 3.67$ & $4.00 \pm 2.19$ & 0.882 \\
\hline Poor DM II management (n \%) & $6(30.00 \%)$ & $4(66.67 \%)$ & 0.105 \\
\hline Underlying diseases (n \%) & $11(55.00 \%)$ & $4(66.67 \%)$ & 0.612 \\
\hline \multicolumn{4}{|l|}{ Clinical parameters } \\
\hline Fever (n \%) & $15(75.00 \%)$ & $6(100 \%)$ & 0.671 \\
\hline Headache (n \%) & $19(73.08 \%)$ & $6(100 \%)$ & 0.419 \\
\hline Nausea/Nomiting (n \%) & $10(50.00 \%)$ & $6(100 \%)$ & $0.027^{*}$ \\
\hline Vision disorders (n \%) & $3(15.00 \%)$ & $1(16.67 \%)$ & 0.921 \\
\hline Auditory symptoms (n \%) & $3(15.00 \%)$ & $1(16.67 \%)$ & 0.921 \\
\hline Seizure (n \%) & $2(10.00 \%)$ & $1(16.67 \%)$ & 0.654 \\
\hline Conscious disturbance (n \%) & $5(25.00 \%)$ & $3(50.00 \%)$ & 0.245 \\
\hline \multicolumn{4}{|l|}{ CSF examinations } \\
\hline Opening pressure $\left(\mathrm{mmH}_{2} \mathrm{O}\right)$ & $258.00 \pm 99.92$ & $316.67 \pm 98.20$ & 0.295 \\
\hline CSF protein (mg/l) & $983.35 \pm 641.03$ & $1175.20 \pm 411.96$ & 0.268 \\
\hline CSF glucose (mmol/l) & $2.38 \pm 1.13$ & $1.95 \pm 0.40$ & 0.882 \\
\hline Brain images abnormality ${ }^{a}(n \%)$ & 13 (65.00\%) & $5(83.33 \%)$ & 0.393 \\
\hline
\end{tabular}

Abbreviations: CM cryptococcal meningitis, DM // type II diabetes mellitus, CSF cerebrospinal fluid

${ }^{a}$ Brain image abnormalities included meningeal enhancement, hydrocephalus, cerebral infarction and so on ${ }^{*} p<0.05$ 
Cryptococcal meningitis patients with type II diabetes mellitus showed higher mortality compared with nontype II diabetes mellitus group. In the type II diabetes mellitus group, $73.07 \%$ of patients were $>60$ years old, and as the prevalence of diabetes mellitus also increases in the aged population, age-associated reductions in functional immune status may be a risk factor for mortality [28]. As shown in Table 3, the presentation of nausea and vomiting was a possible risk factor for mortality type II diabetes mellitus patients. The presence of nausea and vomiting may indicates patients with higher opening pressure, which has been reported to be associated with poor prognosis [24]. We found that $50 \%$ of type II diabetes mellitus patients had a more than 5-year history of type II diabetes mellitus, and $38 \%$ of type II diabetes mellitus patients $(n=10)$ were reported to be untreated or had poor control of blood glucose despite treatment. We also found that more than half of the patients who died had a history of uncontrolled or poorly controlled blood glucose levels, indicating that blood glucose management may be a probable cause of the poor prognosis of cryptococcal meningitis, as shown in previous studies [29-31].

There are several limitations of this study. First, this was a retrospective study performed in a single hospital by reviewing medical records. Secondly, we could not appropriately assess the impact of voriconazole therapy on mortality, which requires dedicated studies with large patient numbers. Thirdly, according to the different serotypes of Cryptococcus, its manifestations and prognosis are different, but this was not further assessed in this study. Further large prospective multicenter studies are needed to improve the generalizability of the data on cryptococcal meningitis .

\section{Conclusion}

In conclusion, our study demonstrated that cryptococcal meningitis patients with type II diabetes mellitus differ significantly from patients without type II diabetes mellitus with respect to clinical symptoms such as visual disorders and cerebrospinal fluid protein levels. In addition, it is necessary to note that type II diabetes mellitus patients experienced higher opening pressure, higher glucose and longer hospitalization than non- type II diabetes patients. Our data also suggested that nausea and vomiting could be related to mortality in patients with type II diabetes mellitus. We need to highlight the importance of type II diabetes mellitus management, considering that the majority of cryptococcal meningitis patients with type II diabetes mellitus who died had poorly controlled blood glucose levels.

\section{Abbreviations}

CM: Cryptococcal meningitis; DM II: Type II diabetes mellitus; Cl: Confidence interval; CSF: Cerebrospinal fluid; IICP: Increased intracranial pressure;
$C T$ : Computed tomography; MRI: Magnetic resonance imaging; AMB: Amphotericin B; 5-FC: Flucytosine; lip AMB: Lipid amphotericin B infusion; FCZ: Fluconazole; VCZ: Voriconazole

\section{Acknowledgements}

Not applicable.

\section{Authors' contributions}

L.H.L.X.J., and Z.L. contributed to study design and the writing of the paper. F.W.J, and Z.K.M. contributed to methodology. A.A.I.M.,H.D.Y. and C.X.Z. had access to take responsibility for data collection. W.H.W.L.L.Y.L.J.J., and X.B has helped with data analysis. L.W.Q.P.W.H., and Z.Q.L. contributed to study design and modified the manuscript for final submission. All authors read and approved the final manuscript.

\section{Funding}

This work was supported by the Major National R\&D Projects of the National Health Department (2018ZX10101003), National Natural Science Foundation of China (82072257), Shanghai Science and Technology Committee (grant numbers 17DZ2272900), Shanghai Municipal Commission of Health and Family Planning (2017ZZ01024-001), the Shanghai Sailing Program (19YF1448000), and Chinese Academy of Engineering (2019-XY-33, 19-HNXZ-06 and 2020-XY-61-01). The funders had no role in the study design, data collection and analysis, decision to publish, and preparation of the manuscript.

\section{Availability of data and materials}

The datasets used and analyzed during the current study are available from the corresponding author on reasonable request.

\section{Ethics approval and consent to participate}

The study was approved by the ethics committee of the Jiangxi Chest Hospital (no. 20190304). Informed consent was waived by the Institutional Review Board due to the retrospective nature of the study design.

Consent for publication

Not applicable.

\section{Competing interests}

The authors declare that they have no competing interests.

\section{Author details}

'Shanghai Key Laboratory of Molecular Medical Mycology, Shanghai Changzheng Hospital, Second Military Medical University, Shanghai 200003, P. R. China. ${ }^{2}$ Department of Dermatology, Shanghai Changzheng Hospital, Second Military Medical University, Shanghai 200003, P. R. China.

${ }^{3}$ Department of Dermatology, Affiliated Hospital of Hebei University of Engineering, Handan, Hebei, P. R. China. ${ }^{4}$ Westerdijk Fungal Biodiversity Institute, Utrecht, The Netherlands. ${ }^{5}$ Faculty of Medicine, Çukurova University, Adana, Turkey. ${ }^{6}$ Department of Laboratory Medicine, Pinghu Second People's Hospital, Zhejiang, P. R. China. ${ }^{7}$ Department of Clinical Laboratory, The Affiliated Xiangshan Hospital of Wenzhou Medical University, Ningbo, Zhejiang, P. R. China. ${ }^{8}$ Department of Neurology, Jiangxi Chest Hospital, Jiangxi 330000 , P. R. China. ${ }^{9}$ Department of Neurology, Jiangxi Provincial people's Hospital, Nanchang 330006, P. R. China.

Received: 5 July 2020 Accepted: 3 February 2021

Published online: 27 February 2021

\section{References}

1. Hagen F, Khayhan K, Theelen B, et al. Recognition of seven species in the Cryptococcus gattii/Cryptococcus neoformans species complex. Fungal Genet Biol. 2015;78:16-48.

2. Rajasingham R, Smith RM, Park BJ, et al. Global burden of disease of HIVassociated cryptococcal meningitis: an updated analysis. Lancet Infect Dis. 2017:17:873-81.

3. Williamson PR, Jarvis JN, Panackal AA, et al. Cryptococcal meningitis: epidemiology, immunology, diagnosis and therapy. Nat Rev Neurol. 2017;13: $13-24$.

4. Fang W, Zhang L, Liu J, et al. Tuberculosis/cryptococcosis co-infection in China between 1965 and 2016. Emerg microbes infect. 2017;6:e73. 
5. Fang W, Chen M, Liu J, et al. Cryptococcal meningitis in systemic lupus erythematosus patients: pooled analysis and systematic review. Emerg Microbes Infect. 2016;5:e95.

6. Li Y, Fang W, Jiang W, et al. Cryptococcosis in patients with diabetes mellitus II in mainland China: 1993-2015. Mycoses. 2017;60:706-13.

7. Pyrgos V, Seitz AE, Steiner CA, Prevots DR, Williamson PR. Epidemiology of Cryptococcal meningitis in the US: 1997-2009. PLoS One. 2013;8:e56269.

8. Yuchong C, Fubin C, Jianghan C, et al. Cryptococcosis in China (1985-2010): review of cases from Chinese database. Mycopathologia. 2012;173:329-35.

9. Barragan NC, Sorvillo F, Kuo T. Cryptococcosis-related deaths and associated medical conditions in the United States, 2000-2010. Mycoses. 2014;57:741-6.

10. Jongwutiwes $U$, Sungkanuparph S, Kiertiburanakul S. Comparison of clinical features and survival between cryptococcosis in human immunodeficiency virus (HIV)-positive and HIV-negative patients. Jpn J Infect Dis. 2008:61:111-5.

11. Hu Z, Yang Y, Cheng J, Cheng C, Chi Y, Wei H. The use of mannitol in HIVinfected patients with symptomatic cryptococcal meningitis. Drug Discov Ther. 2017;10:329-33.

12. Hong N, Chen M, Fang W, et al. Cryptococcosis in HIV-negative patients with renal Dialysis: a retrospective analysis of pooled cases. Mycopathologia. 2017;182:887-96.

13. Chan JC, Zhang Y, Ning G. Diabetes in China: a societal solution for a personal challenge. Lancet Diabetes Endocrinol. 2104;2:969-79.

14. Xu Y, Wang L, He J, et al. Prevalence and control of diabetes in Chinese adults. JAMA. 2013;310:948-59.

15. Gregg EW, Cheng YJ, Srinivasan M, et al. Trends in cause-specific mortality among adults with and without diagnosed diabetes in the USA: an epidemiological analysis of linked national survey and vital statistics data. Lancet. 2018;391:2430-40.

16. Sanitá PV, Zago CE, Pavarina AC, Jorge JH, Machado AL, Vergani CE. Enzymatic activity profile of a Brazilian culture collection of Candida albicans isolated from diabetics and non-diabetics with oral candidiasis. Mycoses. 2014;57:351-7

17. Mirza S, Phelan M, Rimland D, et al. The changing epidemiology of cryptococcosis: an update from population-based active surveillance in 2 large metropolitan areas, 1992-2000. Clin Infect Dis. 2003;36:789-94.

18. Pappas PG. Cryptococcal infections in non-HIV-infected patients. Trans Am Clin Climatol Assoc. 2013;124:61-79.

19. Lin $\mathrm{KH}$, Chen $\mathrm{CM}$, Chen $\mathrm{TL}$, et al. Diabetes mellitus is associated with acquisition and increased mortality in HIV-uninfected patients with cryptococcosis: a population-based study. J Inf Secur. 2016;72:608-14.

20. Lu J, Weng J, Ji N, et al. China guideline for type 2 diabetes 2010 edition. Diabetes Res Clin Pract. 2014;104:1-52.

21. Lee YC, Wang JT, Sun HY, Chen YC. Comparisons of clinical features and mortality of cryptococcal meningitis between patients with and without human immunodeficiency virus infection. J Microbiol Immunol Infect. 2011; 44:338-45.

22. Chen SC, Slavin MA, Heath CH, et al. Clinical manifestations of Cryptococcus gattii infection: determinants of neurological Sequelae and death. Clin Infect Dis. 2012;55:789-98.

23. Unnikrishnan R, Anjana RM, Mohan V. Diabetes mellitus and its complications in India. Nat Rev Endocrinol. 2016;12:357-70.

24. Bicanic T, Brouwer AE, Meintjes $G$, et al. Relationship of cerebrospinal fluid pressure, fungal burden and outcome in patients with cryptococcal meningitis undergoing serial lumbar punctures. AIDS. 2009;23:701-6.

25. Graybill JR, Sobel J, Saag M, et al. Diagnosis and management of increased intracranial pressure in patients with AIDS and cryptococcal meningitis. The NIAID mycoses study group and AIDS cooperative treatment groups. Clin Infect Dis. 2000;30:47-54

26. Yao Y, Zhang JT, Yan B, et al. Voriconazole: a novel treatment option for cryptococcal meningitis. Infect Dis. 2015;47:694-700.

27. Gamaletsou MN, Sipsas NV, Kontoyiannis DP, et al. Successful salvage therapy of refractory HIV-related cryptococcal meningitis with the combination of liposomal amphotericin B, voriconazole, and recombinant interferon- $\gamma$. Diagn Mcrobiol Infect Dis. 2012;74:409-11.

28. Shaw AC, Joshi S, Greenwood H, Panda A, Lord JM. Aging of the innate immune system. Curr Opin Immunol. 2010;22:507-13.

29. Atun R, Davies JI, Gale EAM, et al. Diabetes in sub-Saharan Africa: from clinical care to health policy. Lancet Diabetes Endocrinol. 2017:5:622-67.

30. Davis KL, Wei W, Meyers JL, Kilpatrick BS, Pandya N. Association between different hemoglobin A1c levels and clinical outcomes among elderly nursing home residents with type 2 diabetes mellitus. J Am Dir Assoc. 2014; 15:757-62.

31. Pearson-Stuttard J, Blundell S, Harris T, Cook DG, Critchley J. Diabetes and infection: assessing the association with glycaemic control in populationbased studies. Lancet Diabetes Endocrinol. 2016;4:148-58.

\section{Publisher's Note}

Springer Nature remains neutral with regard to jurisdictional claims in published maps and institutional affiliations.
Ready to submit your research? Choose BMC and benefit from:

- fast, convenient online submission

- thorough peer review by experienced researchers in your field

- rapid publication on acceptance

- support for research data, including large and complex data types

- gold Open Access which fosters wider collaboration and increased citations

- maximum visibility for your research: over $100 \mathrm{M}$ website views per year

At BMC, research is always in progress.

Learn more biomedcentral.com/submissions 